\title{
ON THE CRYPTOECONOMICS EMERGING RESEARCH AREAS
}

\author{
Dominic BUCERZAN \\ "Aurel Vlaicu” University of Arad, Romania \\ dominic@bbcomputer.ro \\ Crina Anina BEJAN \\ "Aurel Vlaicu" University of Arad, Romania \\ ratiu_anina@yahoo.com
}

\begin{abstract}
Today industry, economy and society is reshaped by emergent technologies like "the third web" also known like distributed ledgers technology or blockchain. It seems that the second era of digital economy begun, introducing new concepts like: blockchain, cryptocurrencies, distributed economy and cryptoeconomics. This article aims to propose an image of the actual state of cryptoeconomics, discussing its present and future implications like a research field and proposing several future trends of development.
\end{abstract}

Keywords: Cryptoeconomics, Blockchain, Cryptocurrencies, Decentralized Economy JEL classification: J11, C8, L86

DOI: $10.12948 / \mathrm{ie} 2019.04 .19$

\section{Introduction}

Today industry, society and economy is reshaped by emergent technologies like "the third web" also known for distributed ledgers or blockchain. The development of digital assets had led to the development of a new economy of tokens which recoded in the last year an average of total market capitalization of over 200 billion US Dollars per month [6].

Second era of digital economy starts with blockchain technology which made possible the convergence of four fields: computer science, mathematics, cryptography and behavioral economics [1]. Cryptocurrencies are the first successful use of this model [7].

The development of cryptocurrencies was influenced by some cultural, political and technological factors starting with libertarianism ideology which believe that centralized authorities should be as minimal as possible. The main goal of the libertarian ideology is to protect one's privacy by using cryptography. The average individuals should be able to selectively reveal oneself to the world [9].

\subsection{Before Blockchain}

The first attempt to design a cryptocurrency was DigyCash. It was implemented in 1989 using latest cryptographic developments, namely Blind Signatures Technology. Using this technology users were able to sign online transactions without revealing their identity [9].

Hashcash is a mechanism designed in 1997 to limit email spam and denial of service attacks using a cryptographic hash puzzle and providing a "prof of work". B-Money was developed in 1998 [9], and proposed some concepts that were later adopted by Bitcoin: a prof of work hash function for creating the digital money; a distributed collective ledger, every participant keeping a copy of it so all the users know who owns what; transactions are accomplished by collective bookkeeping and authenticated by hash functions; participants are awarded for their computational work in creating the money; digital signatures were used to sign the transactions and contracts [9]. 
Also the year 1998 is marked by bit gold, which is a mechanism designed for decentralized cryptocurrency systems.

Bitcoin development is marked in 2008 when a person or a group of people using the pseudonym Satoshi Nakamoto published a whitepaper entitled "Bitcoin: A Peer-to-Peer Electronic Cash System" [8]. It is the first successful solution of an anonymous, trustless and decentralized cryptocurrency system that was widely accepted as a digital money and an alternative for traditional currencies [1]. Bitcoin is based on the fallowing concepts: distributed public ledgers, trustless networks, consensus protocol, blind signatures, transparency, prof of work, hash functions and computational power. Bitcoin had been "played for fun" at it's infancy until 2010 when it became a goods trading accepted currency. Generically the way of designing and creating this protocols and manners that governs a blockchain environment are unified under the term of Cryptoeconomics. To understand how Cryptoeconomics work one should understand the technology to which it applies, there for in the next subsections of this paper we aim to picture the main elements of a blockchain system and to classify this technology.

\subsection{Blockchain main components}

According to hyperledger.org, "A blockchain is a peer-to-peer distributed ledger forged by consensus, combined with a system for "smart contracts" and other assistive technologies."

A blockchain environment provides an immutable way to trace transactions. The main concepts used to develop this immutable environment are described as fallows. Distributed Ledgers Technology inspired by accounting procedures, are used to store data transactions between users. The ledgers are shared, public and trusted [1]. Transactions are records of events, cryptographically secured. In a blockchain network transactions are ordered and batched in blocks. They suppose the transfer of any asset or a record of service being provided. Smart contracts allow automatic execution of transactions if some predefined criteria are met. Miners are nodes that have to solve a cryptographic puzzle in order to propose the next block of transactions. Before a block is chained to the network miners have to reach consensus. Consensus mechanisms consists of protocols that ensures that participants agree upon a state of the system as being the true state. The main consensus models used today are: Prof of Work (PoW), Prof of Stake (PoS), Proof of Elapsed Time (PoET), Simplified Byzantine Fault Tolerance (SBFT) and Proof of Authority (PoA).

\subsection{Types of blockchain}

There are three types of blockchain system all of them created and managed differently:

- Public blockchain: is commonly known due to the cryptocurrency environments; is fully public and secured with cryptographic techniques.

- Consortium blockchain: is not fully public and is controlled by preselected nods;

- Private blockchain: is private and the permission for the blockchain is centralized.

Blockchains can be considered a highly specialized databases. Databases can be divided into two main categories: centralized databases and distributed databases. The last category includes distributed ledgers technology which include blockchains [12].

\section{Cryptoeconomics}

Cryptoeconomics represent a way to analyze, incentivize and secure decision for peers in a decentralized system. It may be consider a metrics for the quality of a blockchain system architecture [9]. To understand Cryptoeconomics one should understand the process of decision making. There are two main steps in decision making [9]: according to the rational 
choice theory, every decision of an actor for an action should be the best available alternative in terms of maximizing utility [5]; a decision must be secured, it should be permanent, immutable and should not be altered by others participants.

\subsection{Cryptoeconomics definitions}

Cryptoeconomics is relatively a new research field. Its definition is not yet stabilized and is constantly evolving. The word Cryptoeconomics is formed by the addition between Cryptography and Economics. Cryptography provides protocols and techniques that secures past transactions in a blockchain, making the legers secure and immutable.

While Cryptography and Economics are two research disciplines with long history, the result of their alliance, Cryptoeconomics it is in its infancy as a research field.

Vlad Zamfir, introduced for the first time a definition for Cryptoeconomics: "A formal discipline that studies protocols that govern the production, distribution, and consumption of goods and services in a decentralized digital economy. Cryptoeconomics is a practical science that focuses on the design and characterization of these protocols" [17].

Vitalik Buterin defines Cryptoeconomics "Cryptoeconomics is basically taking economics with those particular constraints and then adding together insights from fields that are fairly close by to the cryptocurrency space-particularly cryptography, information theory, math, and distributed systems, including all of the research around consensus algorithms, hash functions, signatures, zero-knowledge proofs, and what we know about all of those primitive." [14].

Cryptoeconomics can be defined like a multidisciplinary research field that studies and analyze protocols that manage virtual decision making in a decentralized digital economy. These protocols incentivize the participants to make the best decision for a specific goal and ensuring that the decision history is maintained unaltered in a blockchain environment. Cryptoeconomics focuses on the design and characterization of this protocols for systems that have certain desired properties, by using cryptography to prove properties of past messages and economic incentives to encourage desired future properties [11], [14].

\subsection{Technical tools for Cryptoeconomics}

Cryptoeconomics goal is to find ways to prevent inadequate behavior in an environment in which actors are incentivized to make secured decisions that help the greater good. To be feasible in a virtual environment it must be ensured in the first place that good decisions are made and second that they are permanent and immutable [9].

Main technical tools used by cryptoeconomics are discussed as fallows.

\subsubsection{Cryptography}

Cryptography: in general is used to secure information in adversarial environments. In distributed systems like blockchains cryptography ensures the nonrepudiation of a decision. The main cryptographic protocols applied in blockchain networks are: Cryptographic hash functions to prevent changes to transactions, to connect the blocks and to ensure the consensus; Block Header Hash, for consensus; a header block must satisfy the requirements of being below a certain value in the chain; Digital signatures used to prove by one's identity; every physical identity can have one or more virtual representation. Digital signatures are used in the process of authentication of actors; Erasure codes ensure data preservation and security; erasure codes consists of algorithms that by adding some piece of information to some data if parts of the data are lost, the original data can be reconstructed if there are enough information left; Timelocks are encryption algorithms that use easy methods to encrypt the data but which impose longer decryption time; this method suppose the existence of a build-in delay in the process of decryption, which won't be possible to decrease even if parallel computing is used. 
www.conferenceie.ase.ro

\subsubsection{Economics}

Economics is the science that studies the shipment and the use of wealth having the main target maximum result with limited resources. Basically it studies the process of best decision making in a certain scenario. Transposing this in blockchain environment, economics should give a solution regarding the design of a framework that motivates the participants to behave in a certain way. The answer is found in the Game Theory which analyze the way an actor could maximize its profit in a certain restricted environment while competing with other actors [13]. Game Theory stimulates different factors that can influence the decision of actors. These factors include: the action of other actions in the game and the rewords and penalties determined by each outcome. The rewords and penalties are also known as economic incentives. In blockchain environment incentives should change expected participants profit in order to change the participant's decision. In blockchain networks the most common incentives are [13], [14]: Tokens: units of crypto-digital value that can be increased or decreased depending on the behavior of the participant; Privileges: decision making rights that participants can charge for; Security margin: an amount of money or value that every participant should deposit as a guarantee that they will make the decision needed so that specific requirements are satisfied; in chase in which some participants would violate the specified requirements they will lose the guaranteed deposit minimizing their profit.

\subsection{Cryptoeconomics consensus algorithms/models/protocols}

Cryptoeconomics use protocols to define the rules that will determine the validity of transactions and blocks. After the rules are established algorithms are used to develop the steps to fallow in order to accomplish the predefined rules [16].

Consensus models define what happens in conflict situations and which mining nod will publish a new block. The method of agreement must work even in the situation of possible attacks from malicious users [1]. Based on Distributed System Technology a consensus algorithm ensures that the blockchain network achieves Byzantine Fault Tolerance (BFT). This property makes blockchain able to continue operating in a reliable manner even if some of the nodes fail or act maliciously [15].

\section{Blockchain Performance Metrics - starting point of research for Cryptoeconomics}

Blockchain performances can be a starting point of research areas in Cryptoeconomics, due to the fact that Cryptoeconomics provide the protocols and algorithms needed for the system to operate. According to [2] the performances of a blockchain system can be defined by the values of the fallowing metrics: Submission Speed - maximum number of transactions per unit of time allowed for each node and for the entire network; Network Speed - maximum number of transactions that can be validated in the network in a unit of time; Transaction Endurance the average time needed for a transaction to be validated and written on the network; Transaction Fee - how much a user has to pay for a smart contract to be executed or to process a transaction; System Security (can be divided to several sections: Immutability - the cost of an successful attack of rewriting some chains; Denial of Service Attacks resistance; Trust requirements of nodes; Transaction confidentiality; User anonymity); Nods Hardware Costs the minimum cost of the hardware needed in order to become a node and maintenance costs over time as the network scales; Scalability - the trend of system performances in condition of system development: growth of nodes, growth of users, growth of transactions and geographic dispersion; System Development Costs - the cost of infrastructure and maintenance as the system evolves; Adaptability - the architecture of the system should be able to adapt to various environments. 
Another reference point in cryptoeconomics research may be the applicability of the blockchain system to different areas. There are several fields that blockchain technology can emerge major changes [2], [3]: Finance Markets - may be revolutionize by blockchain by replacing the payments system, the intermediaries and the traditional contracts; Real Estate Industry blockchain can store land registry records and public records of land ownership, providing real time access to the stored data and reducing the need of authenticated documents by middle authority; Health Care Industry - blockchain can reform drugs distribution and supply chain and may optimize transaction of private data of patients; Smart Government - a considerable attention in this sector is gained by identity management using blockchain, even though a certain solution is not yet outlined; Artificial Intelligence - this area is close related with smart contracts development; smart contracts combined with artificial intelligence can optimize blockchain technology providing it the ability to function on their own in a semi-autonomous way; Education - blockchain learning can provide an optimized and transparent learning process in which blocks can be packed and placed to the chain by teachers and learning achievements can be viewed like coins [4]; Reputation System - reputation measures the trust that a community grant to one individual; reputation can be evaluated based on one's previously transactions and interactions on the network; Big Data Analytics - blockchain can ensure data originality, can store private important data as it is distributed and secure; also the technology can provide ways to predict and extract pattern behavior for users.

\section{Conclusions}

Cryptoeconomics main targets are to create a virtual environment based on peer-to-peer network that ensures [10]: trusted execution: every participant in the network must be sure that their transaction will be executed; open access: the participants in the network can access and read the code; fast finality: transactions are executed in short time and irreversibly; decentralized control: no third party should control the network or alter the decision making process for their own profit or interests; inexpensive transactions: the transactions should be cheaper than their alternatives fro from the real world; e.g. Bitcoin transactions are cheaper than bank money transfers both from the point of view of time costs and fees.

In a blockchain architecture cryptoeconomics uses economics to incentivize honest decisions and cryptography to secure decided ones. As a research field cryptoeconomics can be defined like the science that analyze decentralized networks with incentives schemes [13].

After the definition of cryptoeconomics will be stabilized we believe that the future research area will include the studies regarding but not limited to: metrics for cryptoeconomics, blockchain testing; the optimization of consensus protocols; the accuracy of time stamping; scalability of blockchain model; optimization of data availability solutions; privacy and trust of blockchain, distributed consensus and fault tolerance mechanisms; architectures for Applications in e-Governance, Big Data, Cloud and Edge Computing and Internet of Things.

\section{References}

[1] D. Bucerzan, C.A. Bejan, "Blockchain. Today Applicability and Implications" accepted for publication in Proceedings of the 8th edition of International Workshop on Soft Computing Applications, presented at The 8th edition of International Workshop on Soft Computing Applications, September 2018, Arad, Romania.

[2] H. Kakavand, N. Kost De Sevres and C. Bart, "The Blockchain Revolution: An Analysis of Regulation and Technology Related to Distributed Ledger Technologies", January 2017, Available at SSRN: https://ssrn.com/abstract=2849251 
www.conferenceie.ase.ro

[3] Z. Zheng, S. Xie, H.N. Dai, X. Chen and H. Wang, "Blockchain Challenges and Opportunities: A Survey,” Int. J. Web and Grid Services, Inderscience Enterprises Ltd., 2017

[4] C. Holotescu, "Understanding Blockchain Opportunities and Challenges," in Proc. of the 14th International Scientific Conference eLearning and Software for Education Bucharest, 2018.

[5] D. Klein, J. Marx, S. Scheller, Rational Choice and Asymmetric Learning in Iterated Social Interactions - Some Lessons from Agent-Based Modeling. In: Marker K., Schmitt A., Sirsch J. (eds) Demokratie und Entscheidung. Springer VS, Wiesbaden, 2019, doi: https://doi.org/10.1007/978-3-658-24529-0_18, ISBN: 978-3-658-24528-3, pp. 277-294

[6] ***, Global Charts. Total Market Capitalization, Internet: https://coinmarketcap.com/ charts/ [Mar. 13, 2019].

[7] R. Lin and G. Wang, Bitcoin Protocol \& Consensus: A High Level Overview, Blockchain at Berkley, Internet: https://prod-edxapp.edx-cdn block/Week_1_Slides.pdf [Mar. 13, 2019].

[8] S. Nakamoto, Bitcoin: A Peer-to-Peer Electronic Cash System, Internet: https:/ /bitco in. org/bitcoin.pdf [Mar. 13, 2019].

[9] R. Lin and G. Wang, Blockchain History: From Cypherpunks to Jp Morgan Chase, Blockchain at Berkley, Internet: https://prod-edxapp.edx-cdn.org /assets /courseware /v1/303 +2 T2018+ type@ asset+b lock/Week_2_Slides.pdf [Mar. 13, 2019].

[10] A. Nguyen, Intro to Cryptoeconomics, Sept.2018, Internet: https://hackernoon.com/int roto-cryptoeconomics-c984b020d186, [Mar. 13, 2019]

[11] R. Deol, Fundamentals Of Cryptoeconomics: Guide To The Token Economy, online course, B21 Block, Internet: https://www.udemy.com/cryptoeconomics/, [Mar. 13, 2019]

[12] R. Lin and G. Wang, Blockchain History: From Cypherpunks to Jp Morgan Chase, Blockchain at Berkley, Internet: https://prod-edxapp.edx-cdn .org/ assets /cou rsew are/ v1/a98 81x+2 T20 18+ty pe@a sset+ block/Week_3_Slides.pdf, [Mar. 13, 2019].

[13] ***, BerkeleyX: CS198.2x, Blockchain Technology, Internet: https://c our ses .e dx.org/courses/course-v1:BerkeleyX+CS198.2x+3T2018/courseware/74b8, [Mar. 14, 2019].

[14] V. Buterin, Introduction to Cryptoeconomics, Internet: https://.ca/files/introcryptoec onomics.pdf, [Mar. 14, 2019].

[15] ***, Byzantine Fault Tolerance Explained, Internet https://www. binance.vision/ blockchain/byzantine-fault-tolerance-explained, [Mar. 14, 2019].

[16] ***, What Is a Blockchain Consensus Algorithm? Internet: https://www.binance. vision/blockchain/what-is-a-blockchain-consensus-algorithm, [Mar. 14, 2019].

[17] ***, What is Cryptoeconomics? The Ultimate Beginners Guide, Internet: https://bloc kgeeks.com/guides/what-is-cryptoeconomics/ [Mar. 14, 2019]. 